\title{
Perfil profesional de los graduados de Lengua y Literatura Hispánicas de la Facultad de Educación e Idiomas de la UNAN-Managua, desde su propia
} perspectiva

Nora Mercedes Cuadra Baquedano Universidad Nacional Autónoma de Nicaragua, Managua norcedes@yahoo.com

(C) UNAN-Managua Recibido: mayo 2017 Aprobado: junio 2017

\section{RESUMEN}

Este artículo presenta una sección de la investigación titulada: Desempeño y satisfacción laboral de los graduados de la carrera de Lengua y Literatura Hispánicas de la Facultad de Educación e Idiomas de la Universidad Nacional Autónoma de Nicaragua, Managua (2009-2013). El objeto del estudio es analizar la valoración que realizan los graduados y empleadores sobre la satisfacción, el desempeño laboral y el perfil profesional de los graduados, sin embargo, este artículo sólo ofrece los resultados referidos al perfil profesional de la población en estudio, la cual valora muy positivo su perfil profesional por considerarse que está en correspondencia con su desempeño laboral.

\section{SUMMARY}

This article presents a section of the research entitled: Performance and job satisfaction of graduates of the Hispanic Language and Literature degree of the Faculty of Education and Languages of the National Autonomous University of Nicaragua, Managua (2009-2013). The purpose of the study is to analyze the assessment made by graduates and employers on the satisfaction, work performance and professional profile of graduates, however, this article only offers the results referring to the professional profile of the population under study, which Your professional profile is very positive because it is considered to be in correspondence with your work performance.

\section{INTRODUCCIÓN}

La investigación que a continuación se presenta, surge por el interés de conocer la problemática de los graduados de Lengua y Literatura Hispánicas del Departamento de Español de la Facultad de Educación e Idiomas de la UNAN-Managua, ya que los estudios de seguimiento a graduados son una fuente importante de información para propósitos de mejoramiento institucional. Y, en particular, son un insumo fundamental para la autoevaluación institucional y para los procesos de acreditación, pues brindan a las instituciones de educación superior, la oportunidad de analizar las características profesionales de sus egresados y de esta manera reorientar los programas de estudio en términos de pertinencia, para garantizar mejores oportunidades en el mercado laboral a quienes se gradúan.

La temática de seguimiento a graduados es amplia, en este artículo el estudio se limita a los resultados encontrados respecto al perfil profesional de los graduados de la carrera de Lengua y Literatura Hispánicas de la Facultad de Educación e Idiomas de la Universidad Nacional Autónoma de Nicaragua, Managua. 
El tema de seguimiento a graduados es de interés porque las universidades son instituciones formadoras de profesionales que se insertan a un campo laboral que demanda de ellos, no solo la actualización constante de los conocimientos aprendidos, sino también la aplicación de competencias, las cuales deben ser evaluadas tanto por el empleador como por la universidad que los graduó, con el objetivo de verificar si los profesionales graduados responden a las necesidades de la sociedad y desempeñan eficientemente su función y de esta manera comprobar la coherencia entre el perfil de egreso de los graduados y la práctica profesional de los mismos.

Otro aspecto importante de este estudio es que, con la entrada en vigencia de la ley 704 (Ley creadora del Sistema Nacional para el aseguramiento de la calidad de la educación y reguladora del Consejo Nacional de Evaluación y Acreditación), las universidades nicaragüenses se están enfocando con más ahínco en el aseguramiento de calidad para satisfacer las necesidades, tanto de sus estudiantes como de la sociedad, respecto al mercado laboral, y una manera de medir la calidad de estas instituciones es el desempeño de sus graduados en relación con la situación laboral y desempeño profesional. Dicha ley, en su artículo 11, establece que las "Instituciones de Educación Superior, públicas y privadas legalmente establecidas en el país, desarrollarán procesos de autoevaluación institucional, a fin de identificar sus fortalezas y debilidades..."

Esto no es válido solo para Nicaragua sino que,desde hace varios años se ha venido fortaleciendo a nivel internacional la tendencia de evaluación de la actividad universitaria, como una forma de rendición de cuentas a la sociedad y a los gobiernos. En ese contexto de evaluación, el seguimiento de egresados es un asunto de vital importancia para las universidades, ya que el desempeño profesional y personal de los egresados permite establecer indicadores con respecto a la calidad y eficiencia de las instituciones de educación superior. (Asociación COLUMBUS, citado por Vega et.al. 2012, p. 31)

Estos procesos de acreditación están exigiendo la realización de estudios de seguimiento de graduados por carrera específica, por lo tanto, las instituciones de educación superior requerirán de personal experto en el área de seguimiento a graduados para tener un referente de la calidad de la enseñanza brindada a sus graduados, en aspectos tales como los insumos o condiciones de funcionamiento, los procesos, los productos y resultados.

Estas investigaciones, además permiten evaluar las necesidades de los egresados y de esta manera mejorar su formación a través de cursos de posgrado. Por otro lado, puede propiciar una mejor comunicación entre la institución, los egresados y los empleadores.

Según la revisión bibliográfica efectuada para llevar a cabo esta investigación, los estudios de seguimiento a graduados, han beneficiado a las instituciones de educación superior que los han realizado, porque han contribuido a:

- Desarrollar sus programas basados en las exigencias del contexto socioeconómico en el que se desenvolverán sus egresados, de manera que responden a las necesidades socieconómicas actuales.

- Conocer el proceso de transición de la universidad al mercado laboral para valorar la calidad de dicha inserción y cuáles son los factores que favorecen o dificultan dicha inserción y así retroalimentar los procesos formativos. 


\section{Nora Mercedes}

Perfil profesional de los graduados... pp. 41-55

- Evaluar los planes formativos de las carreras.

- Conocer la realidad laboral de sus egresados.

- Establecer una relación entre la universidad y el mercado laboral y mejorar la calidad de la oferta educativa para dotar al estudiante de mejores y mayores recursos para enfrentarse a la vida laboral y convertirse en profesionales agentes de cambio en la sociedad.

- Unificar las perspectivas desde las cuales se realizan los estudios de seguimiento a graduados.

- Realizar una evaluación continua y sistemática de la institución universitaria para innovar, descubrir nuevos métodos de enseñanza-aprendizaje y optimizar recursos.

- Conocer información acerca del desempeño profesional, opiniones y sugerencias sobre la calidad de la educación recibida y de las nuevas demandas del mercado laboral y del medio social.

- Identificar en qué medida se están alcanzando los fines de la educación y si los objetivos institucionales y curriculares se cumplen.

- Conocer el proceso y resultado de la inversión social y económica que realiza el país al formar profesionales

La investigación Desempeño y satisfacción laboral de los graduados de la carrera de Lengua y Literatura Hispánicas de la Facultad de Educación e Idiomas de la Universidad Nacional Autónoma de Nicaragua, Managua, entre el 2009-2013, tiene como objetivo analizar la valoración que realizan los graduados y empleadores sobre la satisfacción, el desempeño laboral y el perfil profesional de los graduados de la carrera de Lengua y Literatura Hispánicas. Este estudio se inicia en un contexto en el que, por mandato de la Ley 704 (Ley creadora del sistema nacional para el aseguramiento de la calidad de la educación y reguladora del Consejo Nacional de Evaluación y Acreditación), las instituciones de educación superior de Nicaragua están llevando a cabo su proceso de autoevaluación institucional, en el que no solo se consideran factores internos sino también externos que ponen en evidencia la calidad de los profesionales graduados en estas instituciones.

\section{MATERIAL Y MÉTODO}

Esta investigación se enmarca en un enfoque multimétodo, adscrita dentro del paradigma sociocrítico, pues no se adhiere solo al reduccionismo de datos (positivismo) ni es meramente una recopilación de datos para verificar teorías preconcebidas, pues como afirma Ricoy (2006), "se aspira desde el paradigma crítico a la unión entre la teoría y la práctica, usando la primera como fundamentación y guía de la segunda, con una interacción mutua" (p. 18).

Según la profundidad u objetivo, esta investigación es de tipo correlacional, de acuerdo con Hernández, Fernández y Baptista, los estudios correlaciónales "... tienen como finalidad conocer la relación o grado de asociación que existe entre dos o más conceptos, categorías o variables en un contexto particular" (2010, p. 81). 


\section{Revista Lengua y Literatura. Vol.3, núm. 1, enero-junio 2017}

Se establecerán relaciones entre variables para identificar sentido de causalidad: significativa o no, rango de interacción entre las variables. Esta relación entre variables permitirá describir en mayor profundidad las características de la población en estudio.

Según el alcance temporal, este estudio es transversal (seccional o sincrónica) porque se estudiará a la población graduada de la carrera de Lengua y Literatura Hispánicas durante los años 2009-2013. Según Beltrán, del Rincón y Arnal, "este tipo de investigación estudia un aspecto de desarrollo de los sujetos en un momento dado" (2003, p. 44).

\section{Sujetos}

El universo del estudio está compuesto por todos los graduados entre el 2009-2013 de carrera de Lengua y Literatura Hispánicas de la Facultad de Educación e Idiomas de la UNAN-Managua que, según datos que lleva la Dirección de Registro Académico, Estadístico y Estudiantil, son 174. Las características de esta población son las siguientes:

- Pueden estar desempleados

- Cursaron sus estudios en el turno regular o sabatino

- Ya tramitaron su título

- Realizan trabajo relacionado o no con la labor docente

Se seleccionó una muestra probabilística estratificada por año de graduación, donde estuvieran representados los graduados de los diferentes años, de manera que fuera proporcional.

Se considera que la muestra de este estudio es representativa de la población porque es un reflejo de ella, es decir, reúne las características principales de la población en relación con la variable en estudio. Además, los elementos de $\mathrm{N}$ se dividieron en varios estratos, de tal forma que, como afirma Ludewing (s.f.), cada estrato formado es internamente homogéneo porque sus integrantes se parecen mucho entre sí en lo que se refiere a la variable a estudiar, mientras que los estratos difieren unos de otros.

Como resultado de la aplicación de la fórmula estadística, la muestra del estudio quedó conformada por 120 graduados.

\section{Instrumento}

En esta investigación se aplicaron: técnicas de investigación documental y técnicas para la obtención de información en el campo.

En el caso de la primera, la revisión bibliográfica se realizó tanto en fuentes directas como indirectas, en físico, bases de datos, tesis, artículos de revista, fuentes electrónicas situadas en la red web, y ha sido la base de la fundamentación teórica y metodológica.

Para la obtención de información en el campo, se utilizó como instrumento el cuestionario (encuesta), el cual se diseñó con preguntas cerradas (escala Likert), utilizando categorías excluyentes, nominales e ítems de escala, donde se les pide que valoren su desempeño laboral. Para determinar el número de preguntas se tomó como referencia la tabla de definición de variables. 


\section{Procedimientos}

Para proceder a la aplicación de la encuesta, se les informó vía telefónica a los graduados sobre el estudio que se estaba realizando y se les pidió su colaboración en el llenado de la encuesta. A los que contaban con una dirección de correo se les envió la encuesta y a los que no, se le hizo una visita a su domicilio.

Una vez recogidos los cuestionarios, se procedió al análisis y discusión de los resultados con la ayuda del programa SPSS V 21, mediante el cual se calcularon estadísticos descriptivos como frecuencias absolutas de cada categoría para descartar la presencia de valores ajenos a los valores originales de cada variable. Luego se procedió al análisis de datos con la elaboración de gráficos, que permitieron realizar un análisis descriptivo de la muestra, atendiendo a los diferentes apartados en que se encuentra estructurado el cuestionario.

\section{RESULTADOS}

Para conocer cómo valoran los graduados el perfil profesional que alcanzaron durante sus estudios en la carrera de Lengua y Literatura Hispánicas, se redactó la siguiente pregunta directriz: ¿Cómo consideran los graduados la educación recibida durante su formación

$$
n=\frac{Z^{2 *} p^{\star} q^{\star} N}{N^{\star} e^{2}+Z^{2 \star} p^{\star} q}
$$

universitaria respecto a los requerimientos en su área laboral?, para obtener respuesta se redactaron 11 ítems (39-49), que se encuentran el bloque D del cuestionario a graduados. A continuación, se presentan los resultados de la encuesta.

\section{Tengo habilidades para la comunicación oral y escrita}

La respuesta a esta pregunta es independiente de si el encuestado trabaja o no, sin embargo, un $5 \%$ respondió que no aplica, lo que puede interpretarse como que la pregunta no fue entendida por este pequeño porcentaje de la muestra. El $71 \%$ responde que está de acuerdo, un $26 \%$ muy de acuerdo y un $3 \%$ en desacuerdo. En la siguiente figura se aprecian estos resultados.

\section{Habilidades parala comunicación}

Muy de acuerdo

De acuerdo
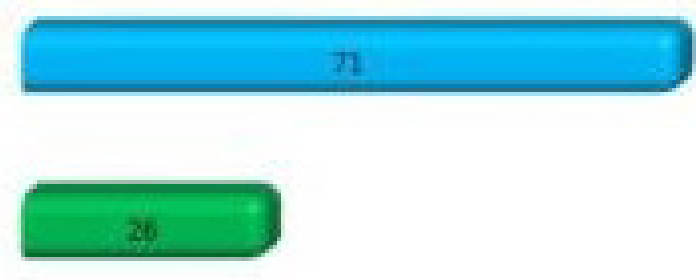

No aplica

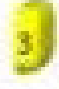


Revista Lengua y Literatura. Vol.3, núm. 1, enero-junio 2017|

Poseo capacidad para coordinar equipos de trabajo (en el aula o a nivel de compañeros)

Los datos de la figura 2 revelan que el 97\% de los encuestados está de muy acuerdo y de acuerdo en que posee capacidad para coordinar equipos de trabajo. Solo $3 \%$ no aplica a esta pregunta.

Este último porcentaje está en correspondencia con la población en estudio que se encuentra desempleada.

\section{Coordinación de equipos de trabajo}

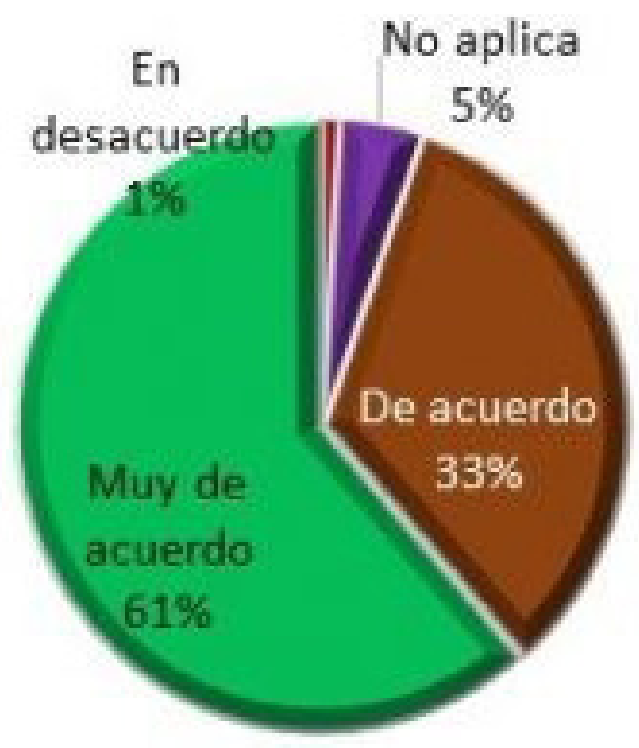

Tengo conocimiento y dominio de la teoría especializada, didáctica y pedagógica para desarrollar mi trabajo

Una parte significativa de los encuestados está muy de acuerdo y de acuerdo (59\% y $38 \%$ ) en que tienen conocimiento y dominio de la teoría especializada, didáctica y pedagógica para desarrollar su trabajo, frente a un $1 \%$ que está en desacuerdo. Un $2 \%$ responde que la pregunta no aplica a su situación.

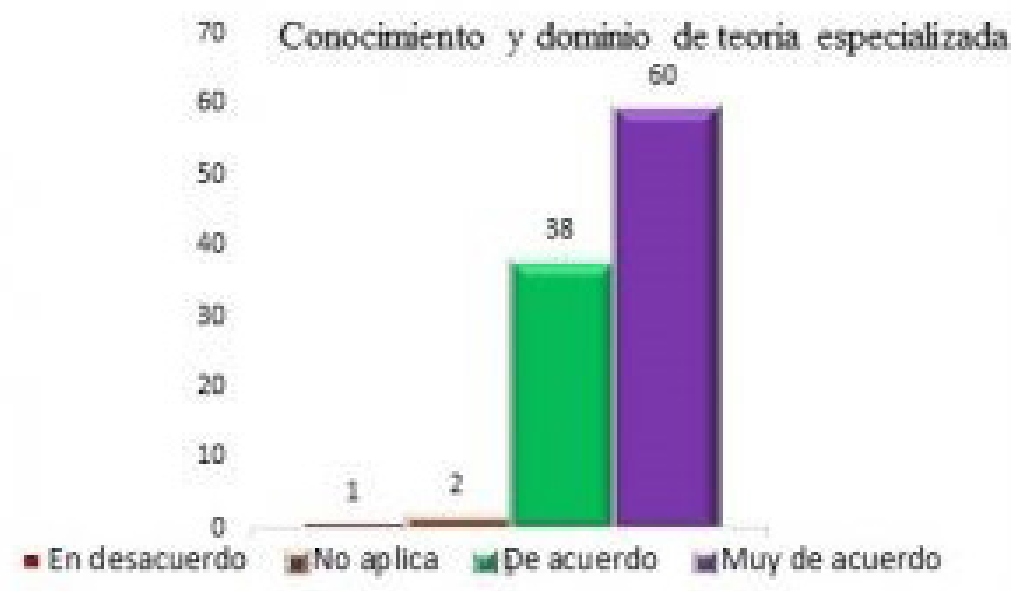


Poseo conocimiento y dominio de la metodología curricular para orientar acciones educativas

Del total de respuestas ante la pregunta de si posee conocimiento y dominio de la metodología curricular para orientar acciones educativas, en la figura 4 se puede apreciar que la inmensa mayoría (49\% y 43\%) expresa una opinión favorable con de acuerdo y muy de acuerdo, respectivamente. El restante $8 \%$ se divide en no responde y no aplica.

\section{Conocimiento $y$ donunio de metodologia} especializada

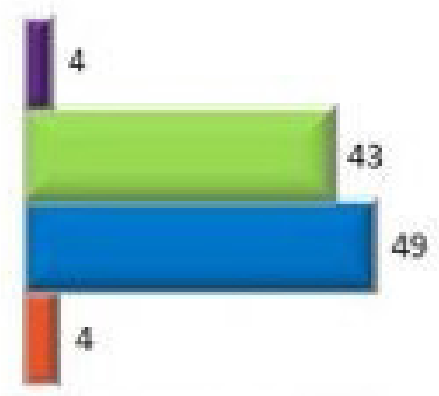

No responde aMuy de acuerdo aDe acuerdo = No aplica

Poseo habilidades para buscar información pertinente y actualizada para el desarrollo de mi trabajo

El desarrollo de una profesión exige de la constante búsqueda de información pertinente, en este aspecto, la proporción más alta de los encuestados (64\% y $29 \%$ ) expresan estar muy de acuerdo y de acuerdo en que tienen esa habilidad, la restante proporción responde que no aplica la pregunta y tan solo un $1 \%$ no respondió, como se aprecia en la figura 5.

\section{Habihidades para buscar información pertinente $y$ actuahizada}

\section{$64 \%$}

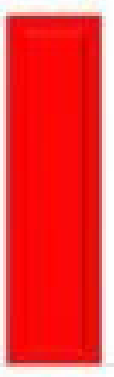

$1 \%$

No aplica De acuerdo Muy de acuerdo No responde 
Revista Lengua y Literatura. Vol.3, núm. 1, enero-junio 2017|

\section{Tengo habilidades para aprender nuevos conocimientos}

Otra habilidad importante es aprender nuevos conocimientos que permitan la actualización de los mismos y el porcentaje más alto de los encuestados ( $75 \%$ y $19 \%$ ) manifiesta estar muy de acuerdo y de acuerdo en que poseen esa habilidad, lo que representa un porcentaje significativo del $94 \%$ de respuesta a favor. El $5 \%$ considera que su profesión no le exige tener o aplicar esta habilidad y un $1 \%$ no respondió (ver figura 6 ).

\section{Habilidades para aprender nuevos conocimientos}

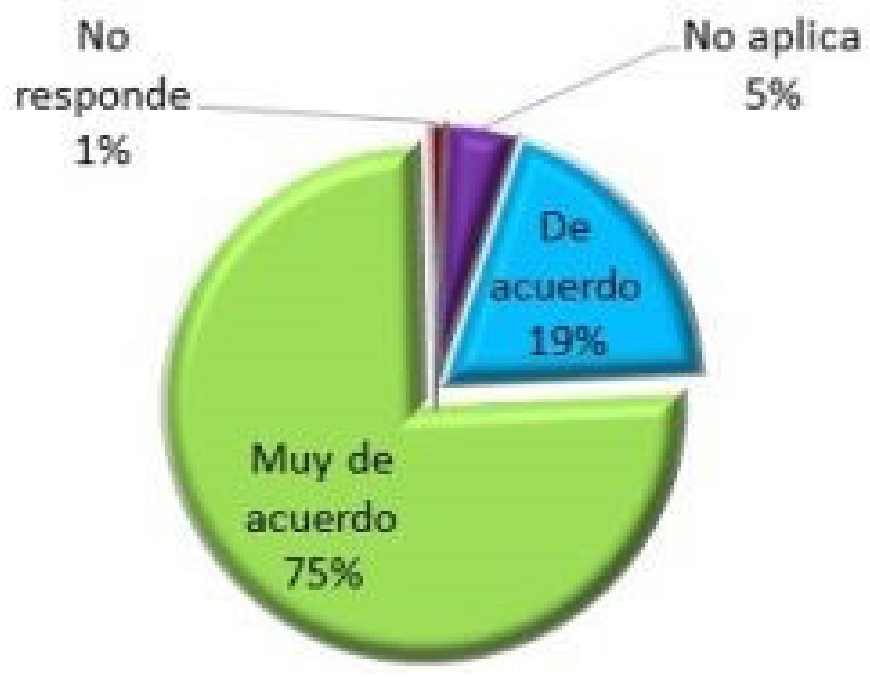

\section{Tengo capacidad para trabajar en equipo}

Respecto a la capacidad para trabajar en equipo, casi la totalidad de los graduados $99 \%$, revela estar muy de acuerdo y de acuerdo con que son capaces de realizar trabajo en equipo, lo que se aprecia en la figura 7.

\section{Capacidad para trabajr en equipo}

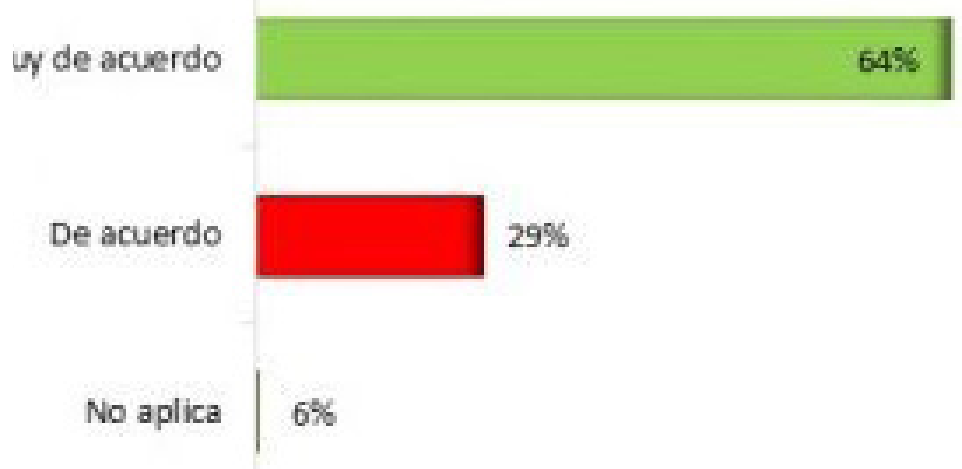




\section{Soy respetuoso de la opinión ajena}

En la figura 8 se aprecia que la inmensa mayoría de los encuestados opina estar muy de acuerdo y de acuerdo en que son respetuosos de la opinión ajena. El $4 \%$ respondió que no aplica.

\section{Respeto de la opinión ajena}

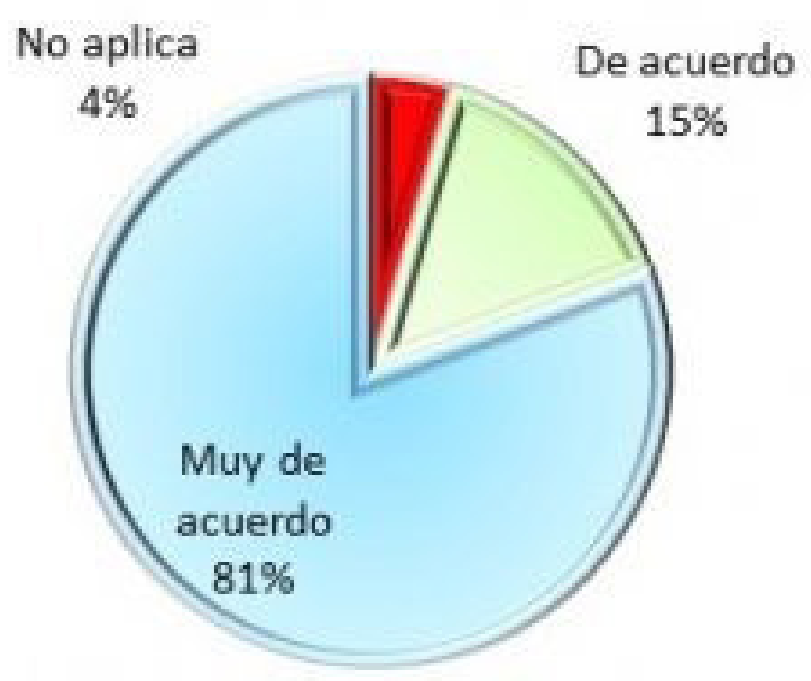

\section{Mi cargo/puesto aemanaa ios conocımıentos que aqquirí durante mi carrera}

Según lo revelado por los encuestados en la pregunta 7 de este cuestionario (cargo o puesto que desempeña), del $96 \%$ que cuenta con empleo, el $90 \%$ se desempeña en el área para la cual fueron preparados, porcentaje que coincide con los resultados obtenidos cuando se les consultó sobre si el cargo demanda los conocimientos adquiridos, pues el $89 \%$ manifiesta estar muy de acuerdo y de acuerdo (71\% y 18\%), una proporción muy baja $3 \%$, está muy en desacuerdo y en desacuerdo (ver figura 9).

\section{Mi cargo demanda los conocimientos que adquiri}

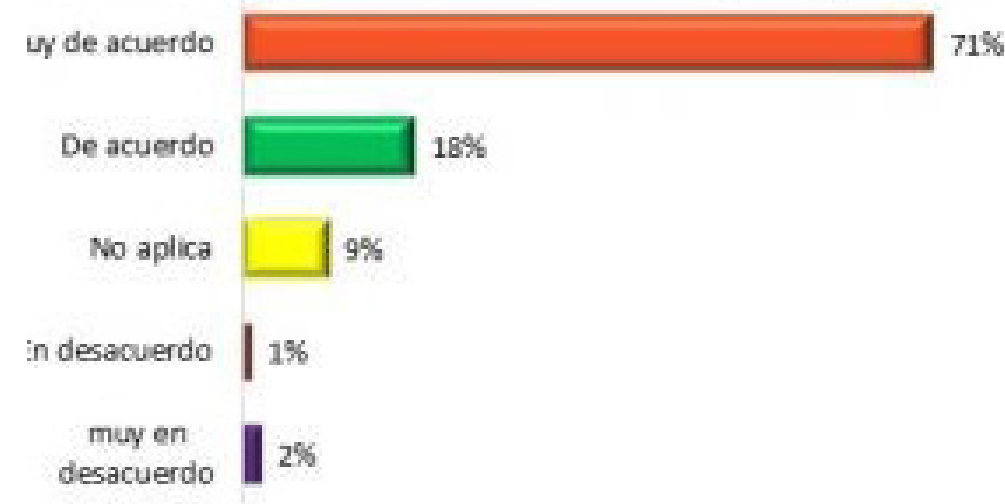


Revista Lengua y Literatura. Vol.3, núm. 1, enero-junio 2017

Los conocimientos adquiridos durante mis estudios universitarios son suficientes para el ejercicio de mi trabajo

Los datos reflejados en la figura 10, revelan que solo una mínima proporción de los encuestados $10 \%$, no aplican en su trabajo los conocimientos adquiridos durante la carrera, por lo que no pueden valorar si son suficientes, en el polo opuesto encontramos a un $90 \%$ que está muy de acuerdo y de acuerdo en que los conocimientos son suficientes para el ejercicio de la carrera.

\section{Conocimientos sufientes para realizar el trabajo}

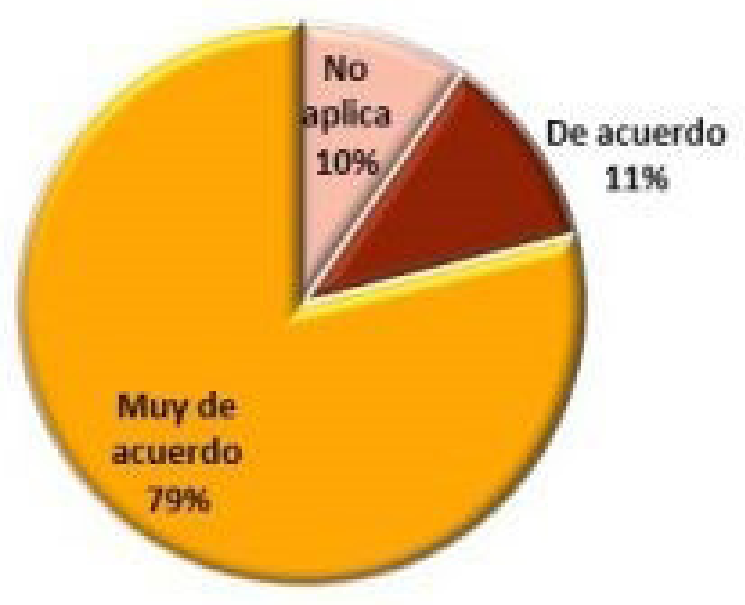

\section{Si tuviera que estudiar nuevamente esta carrera escogería la misma universidad}

Al consultar a los graduados sobre si tuvieran que estudiar nuevamente la carrera de Lengua y Literatura Hispánicas, volverían a estudiar en la UNAN-Managua, la inmensa mayoría (83\%) expresó estar muy de acuerdo, un 8\% de acuerdo, para una opinión favorable del 91\%, frente a un $5 \%$ que respondió estar en desacuerdo, un $4 \%$ no respondió, como se refleja en la figura 11.

Volvria a estudiar en la misma universidad

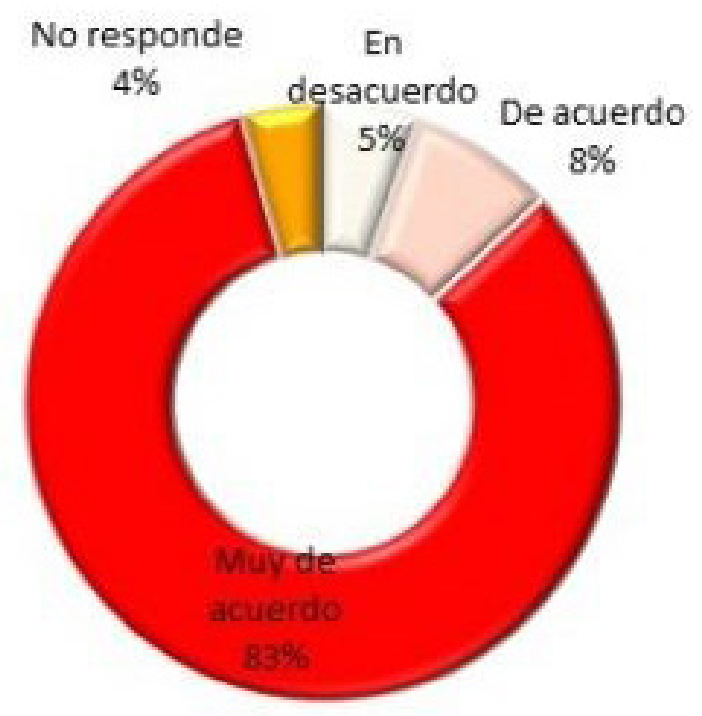




\section{DISCUSIÓN DE LOS RESULTADOS}

En las conclusiones extraídas a la luz de los resultados obtenidos, se plasman los aspectos más relevantes que podrían ser de utilidad para las IES donde se imparte la carrera de Lengua y Literatura Hispánicas. Claro está que estas conclusiones se ajustan a las características concretas de la muestra del estudio, de modo que no pretenden ser completamente generalizables a todos los contextos y ámbitos educativos superiores. Se intentará, a través de las conclusiones, dar respuesta al problema de investigación planteado, sobre el perfil de los graduados de Lengua y Literatura Hispánicas del Departamento de Español de la Facultad de Educación e Idiomas de la UNAN-Managua.

En primer lugar, se abordará la constatación de hipótesis que se plantearon al iniciar este estudio.

Al constatar la hipótesis "El desempeño laboral de los egresados de la carrera de Lengua y Literatura Hispánicas de la Facultad de Educación e Idiomas de la UNAN-Managua se relaciona significativamente con el grado conocimientos adquiridos durante el estudio de la carrera, los resultados indican una relación significativa entre los grupos de variables que se relacionaron. El rendimiento de un individuo en su trabajo, depende en gran medida de su preparación profesional, de acuerdo con la Organización para la Cooperación y el DesarroIlo Económico (OCDE), "[...] el capital humano es el conocimiento, las competencias y otros atributos acuerpados en los individuos y que resultan relevantes a la actividad económica [...]" (citado por Campos, s.f. p. 2). Para Llamas, citado también por Campos, el capital humano es "el acervo de habilidades, destrezas y conocimiento productivo incorporado en las personas.... (s.f. p. 2). Según lo anterior, se puede afirmar que existe correspondencia entre la formación profesional recibida por los graduados y su actividad laboral y que además ha favorecido su desempeño exitoso.

Los grupos de variables que se relacionaron para confirmar esta hipótesis fueron las siguientes:

- Tengo conocimiento y dominio de la teoría especializada didáctica y pedagógica para desarrollar mi trabajo / Los materiales didácticos que selecciono y elaboro favorecen los procesos de enseñanza aprendizaje.

- Constantemente estoy innovando los métodos de trabajo con los discentes / Tengo habilidades para aprender nuevos conocimientos.

- Poseo conocimiento y dominio de la metodología curricular para orientar acciones educativas / Selecciono, elaboro y utilizo materiales didácticos pertinentes con el contexto social y político de los estudiantes.

- Poseo habilidades para buscar información pertinente y actualizada para el desarrollo de mi trabajo / Soy capaz de resolver situaciones problemáticas de mi área de trabajo. 
A partir del análisis general de los datos, se obtienen las siguientes conclusiones:

La tasa de desempleo en Nicaragua es de 12.7\%, según el Servicio Europeo de Acción Exterior, y según el FUNIDES, uno de los sectores con mayor cantidad de empleo es educación, en los resultados de las encuestas encontramos que de la totalidad de los encuestados, el porcentaje más alto (94\%), es decir, 107 , se cuentan con algún tipo de empleo, mientras que solo una mínima proporción (4\%) se encuentran en el desempleo. Esto representa un porcentaje relativamente bajo si consideramos la alta tasa de desempleo del país. Se puede decir que existe demanda de profesionales en el sector educación. Esto también es un indicador de que los graduados de la UNAN-Managua cuentan con un prestigio por su formación profesional y que los empleadores confían en la calidad de quienes estudian en esta alma máter.

La tercera dimensión que se analizó fue el perfil profesional del graduado, de acuerdo con la definición del grupo de variables que componen esta dimensión, el $69 \%$ de los graduados está muy de acuerdo y el $25 \%$ de acuerdo en que su perfil profesional favorece su desempeño laboral, lo que se puede interpretar como que este cumple con el conjunto de rasgos y capacidades que les permite a los graduados realizar las tareas para las que fue capacitado y que además es competente en esa área (Hawes y Corvalán, citados por Cantero, 2012).

Un 94\% de los graduados está muy de acuerdo y de acuerdo en que su perfil profesional lo dota de habilidades y destrezas para ejercer su profesión, si bien es cierto, el MINED no tiene establecido el perfil profesional del docente, en la Ley General de Educación, en el artículo 27 se definen los objetivos generales del programa de formación docente que abarca diferentes áreas: pedagogía, didáctica, conocimiento científico especializado para cada área del saber, valores éticos, morales, sociales, ecológicos y humanos, de manera que estos profesionales desarrollen de forma integral su profesión y contribuyan con el desarrollo de la sociedad. Tanto los graduados como los empleadores coinciden en que el graduado posee no solo conocimientos específicos, sino también cualificaciones relacionadas con las competencias básicas y genéricas: como la capacidad para el trabajo en equipo, habilidades para la negociación, planificación, habilidades para la lectura y escritura, comunicación oral, matemáticas, entre otras (Mertens, citado por Rodríguez, 2007).

En lo relativo a los aspectos que se debe mejorar, existe coincidencia entre las respuestas de empleadores y graduados: Fortalecer y brindar estrategias de enseñanza para la redacción, ortografía, para la comprensión lectora y análisis literario. Fortalecer las asignaturas del área de gramática, didáctica y la investigación (científica, documental, sobre la realidad).

Según los empleadores, los requisitos que la institución estipula para contratar al docente de Lengua y Literatura, no solo contempla conocimientos específicos del área, sino también aptitudes, actitudes y destrezas, buenas relaciones humanas, comunicación fluida, colaboración, disciplina y experiencia. Para que la formación de los profesionales sea pertinente con las exigencias del mercado laboral y garantice altas posibilidades de su inserción laboral, las instituciones de educación superior deben "diseñar un currículo potenciador de competencias profesionales en estrecha combinación con el desarrollo de competencias genéricas, continuar o iniciar estudios de seguimiento de egresados, consultas a los empleadores" (Zapata, 2007, citado por Gutiérrez et al., 2012, p. 157) Según Ginés (2012), "las necesidades del nuevo contexto de la educación superior exigen formar a los individuos en un conjunto amplio de competencias que incluyan los conocimientos, pero también las habilidades y las actitudes que son requeridas en el puesto de trabajo"(p. 202). 


\section{Nora Mercedes Perfil profesional de los graduados... pp. 41-55}

En el caso de los graduados de Lengua y Literatura Hispánicas, otros aspectos que se deben mejorar para garantizar calidad en su formación, destacan: Mejor trato, respeto y actitud positiva de los docentes hacia los estudiantes, mejorar equipos tecnológicos para la enseñanza y manejo de las TIC, más horas de Prácticas Docentes, unificar los programas de la carrera con los del MINED, ofrecer maestrías al alcance de los salarios de los docentes de secundaria, exigir calidad en la ortografía y caligrafía a los estudiantes de Lengua y Literatura, mejor la preparación de los docentes que imparten las clases.

Sobre la base de los resultados encontrados, esta investigación permitirá al Departamento de Español de la Facultad de Educación e Idiomas de la UNAN-Managua:

- Conocer información acerca del desempeño profesional, opiniones y sugerencias acerca de la calidad de la educación recibida y de las nuevas demandas del mercado laboral y del medio social.

- Determinar en qué medida se están alcanzando los fines de la educación y si los objetivos institucionales y curriculares se cumplen.

- Conocer la realidad laboral de sus egresados.

- Promover entre los graduados de Lengua y Literatura Hispánicas los programas de posgrado que se ofrecen en este Departamento Docente, de modo que puedan especializarse en un área y actualizar los conocimientos adquiridos durante la carrera. 
Ley Creadora del Sistema Nacional para el aseguramiento de la calidad de la educación y reguladora del Consejo Nacional de Evaluación y Acreditación. La Gaceta N 172. Managua, Nicaragua, 12 de septiembre de 2011.

Beltrán, A., del Rincón, D. y Arnal, J. (2003). Bases metodológicas de la investigación Educativa. Barcelona, España. Ediciones Experiencia, S.A.

Cantero, M. (2012). Competencias socio-emocionales en la inserción laboral del egresado universitario (Tesis doctoral, Universidad de Alicante, España). Recuperado de https://rua. ua.es/dspace/bitstream/10045/25132/1/Tesis_Cantero_Vicente.pdf

Fundación Nicaragüense para el Desarrollo Económico y Social (FUNIDES). (2015).

Primer Informe de Coyuntura Económica. Recuperado de http://funides.com/wp-content/ uploads/2015/04/primer-informe-de-coyuntura-economica-2015.pdf

Ginés, J. (2012). Educación superior y empleo, resultados y futuro en Europa y América Latina. En Centro Interuniversitario de Desarrollo (CINDA) (Ed.), Seguimiento de egresados e inserción laboral: experiencias universitarias. (pp.200-215). Santiago de Chile: Alfabeta Artes Gráficas Recuperado de www.cinda.cl/.../libros/01\%20Cinda\%20Seguimiento\%20indd\%20 (2).pd.

Gutiérrez, A., Faúndez, F., Castillo, J., Pantoja, L., Audibert, M., Ponce, M., Contreras, S. (2012). Variables que inciden en la inserción Laboral de titulados universitarios. En Centro Interuniversitario de Desarrollo (CINDA) (Ed.), Seguimiento de egresados e inserción laboral: experiencias universitarias. (pp.153-178). Santiago de Chile: Alfabeta Artes Gráficas Recuperado de www.cinda.cl/.../libros/01\%20Cinda\%20Seguimiento\%20indd\%20(2).pd

Hernández, R., Fernández, C. y Baptista, P. (2010). Metodología de la Investigación. México. Mc Graw Hill.

Ludewing, C. (s.f.). Universo y Muestra. Recuperado de http://www.smo.edu.mx/colegiados/ apoyos/muestreo.pdf

Metzner, N. (2012). 2035 -Ahora es el tiempo, ¡vos decidís! Las juventudes de Nicaragua - una oportunidad olvidada Managua. Recuperado de http://www.eduquemos.org.ni/eduquemos/images/publicaciones/foro_educacion_la_llave_maestra_del_progreso/estudio_ de_ddhh_de_la_juventud_empleo_partipacion_y_educacion_mjn.pdf

Oliván, C. (s.f). Empleabilidad y Empleo: formación universitaria y mercado de trabajo en España y Aragón. Recuperado de www.aragon.es/.../140511\%20Empleabilidad\%20y\%20 Empleo.pdf 


\section{- REFERENCIAS -}

Ricoy, C. (2006). Contribución sobre los paradigmas de investigación. Revista do Centro de Educação, 21(1),11-22. Recuperado de http://www.redalyc.org/pdf/1171/117117257002.pdf

Rodríguez, H. (2007). El paradigma de las competencias hacia la educación superior. Revista de la Facultad de Ciencias Económicas, Universidad Militar Nueva Granada. 15(1). Recuperado de http://www.unipamplona.edu.co/unipamplona/portalIG/home_9/recursos/ portal2013/08102015/paradigmas_competencias_.pdf

Vega, A., González, E., Toro, G., Fonseca, G., Sánchez, J., Peña, M., Carrasco, S. (2012). Aspectos referenciales sobre la inserción laboral y seguimiento de egresados. En Centro Interuniversitario de Desarrollo (CINDA) (Ed.), Seguimiento de egresados e inserción laboral: experiencias universitarias. (pp. 19-56). Santiago de Chile: Alfabeta Artes Gráficas Recuperado de http://www.cinda.cl/.../libros/01\%20Cinda\%20Seguimiento\%20indd\%20(2).pdf 